\title{
Canlı vericili karaciğer transplantasyonu öncesi intraoperatif olarak ölçülen greft ağırlığının tahmininde bilgisayarlı tomografinin rolü
}

\author{
Role of computed tomography in estimating graft weight measured intraoperatively before living \\ donor liver transplantation
}

\author{
(DMustafa ÖZDEMIR ${ }^{1}$, (DHalil ÖZER ${ }^{1}$, (DYiğit DÜZKÖYLÜ², (DRıza Sarper ÖKTEN ${ }^{1}$, (DFatma Ayça Edis ÖZDEMIR ${ }^{1}$ \\ Ankara Şehir Hastanesi, ${ }^{1}$ Radyoloji Kliniği, ${ }^{2}$ Gastroenteroloji Cerrahi Kliniği, Ankara
}

Giriş ve Amaç: Bu çalışmanın amacı canlıdan karaciğer transplantasyonunda greftin değerlendirilmesinde bilgisayarlı tomografi ile ölçülen tahmini greft volümünden korreksiyon faktörü kullanılarak hesaplanan tahmini greft ağırlığı ile gerçek greft ağırlığının karşılaştırılması ve bu korreksiyon faktörünün efektivitesinin araştırılmasıdır. Gereç ve Yöntem: Ocak 2009-Temmuz 2015 tarihleri arasında 42 canlı karaciğer donörünün (20 kadın, 22 erkek; ortalama yaş, 42.23 yıl土17.25 (1852) dinamik kontrastlı hepatik bilgisayarlı tomografi kesitlerinden elde edilen veriler retrospektif olarak tarandı. Donörler, karaciğer nakli için karaciğer sağ lob rezeksiyonu yapılan sağlıklı erişkinlerdi. Bilgisayarlı tomografi ile ölçülen tahmini greft volümü (cc) Lemke ve arkadaşlarının formülü (tahmini greft ağırlığı $(g)$ : 0.678 x tahmini greft volümü (cc) +143.704 g) kullanılarak tahmin edildi. Tahmini greft ağırlığı ile gerçek greft ağırlığı arasındaki ilişkiyi saptamak için Pearson korelasyon test kullanıldı. Bulgular: Ameliyat öncesi ortalama karaciğer hacmi 918.265 (580-1230) cc idi. Ameliyat öncesi tahmini greft ağırlığı ve gerçek greft ağırlığı ortalama ağırlıkları sırasıyla 818.119 (550-1171) gr, 790.359 (505-1150) gr ölçüldü. Gerçek greft ağırlığı ile preoperatif tahmini greft volümü arasında istatistiksel bir ilişki yoktu. Ancak gerçek greft ağırlığı ile preoperatif tahmini greft ağırlığı arasında güçlü bir istatistiksel ilişki tespit edildi. Pearson korelasyon analizi sonuçları güçlü bir korelasyon gösterdi ( $r=0.98, p<0.001)$ ve regresyon modelinin sonucu bu bulguyu destekledi ( $p$ <0.0001). Sonuç: Sonuç olarak canlıdan karaciğer naklinde greftin değerlendirilmesinde bilgisayarlı tomografi ile ölçülen volümün gerçek ağırlıktan farklı olduğu, Lemke ve ark.'nın geliştirdiği formülün aktüel ağırlığı tahminde yeterli ve etkili olduğu tespit edildi.

Anahtar kelimeler: Karaciğer nakli, bilgisayarlı tomografi, organ boyutu

\section{GíRiş}

Karaciğer nakli, son dönem karaciğer hastalığı ve erken dönem karaciğer kanserini tedavi etmek için tüm dünyada yaygın olarak uygulanmaktadır (1). Ancak, kadavra donörlerinin yetersizliği, canlı vericili karaciğer naklini zorunlu kılmaktadır (2). Canlı vericili karaciğer naklinin başarısı, sadece donörün cerrahiye uygunluğunu değil aynı zamanda karaciğer steatozunun, vasküler ve biliyer anatominin detaylı preoperatif değerlendirmesini ve ope-
Background and Aims: The aim of our study was to evaluate the graft in an alive donor liver for transplantation using computerized tomography (CT) estimated graft volume, which is calculated by comparing the estimated graft weight with the actual graft weight through a correction factor and its efficacy. Materials and Methods: The dynamic contrast-enhanced hepatic computerized tomography scans of 42 alive liver donors (20 females, 22 males; mean [standard deviation, range] age, 42.23 [17.25, 18-52] years), consecutively registered from January 2009 to July 2015 were retrospectively analyzed in this study. The donors were healthy adults who had undergone resection of the right liver lobe for liver transplantation. The calculated estimated graft volume was compared with the actual intraoperative graft weight of the harvested right hepatic lobe. Results: The mean preoperative liver volume was found to be 918.265 (580-1230) cc. The mean (range) calculated preoperative estimated graft weight and the actual graft weight were 818.119 (550-1171) g and 790.357 (505-1150) g, respectively. No significant association was found between the actual graft weight and the preoperative estimated graft volume. However, there was a strong statistical association between the actual graft weight and preoperative estimated graft weight. The Pearson's correlation analysis revealed a strong correlation $(r=0.98, p<0.001)$, and the result of the regression model supported the finding $(P<0.0001)$. Conclusions: We found that the computerized tomography-estimated graft volume is different from the preoperative actual weight, and the current formula used worldwide is adequate and efficient for predicting the actual weight for evaluating the graft in a living donor liver for transplantation.

Key words: Liver transplantation, tomography, organ size

rasyon sonrası vericide kalacak karaciğer ile alıcıya takılan greftin yetip yetmeyeceğinin değerlendirmesine bağlıdır.

Hacim ve ağırlık anlamında uygun greftin elde edilmesi canlı vericili karaciğer naklinin en önemli kısımlarından biridir. Tam otomatik yazılım sistemleri son yıllarda kullanılmasına rağmen, manuel hacimsel hesaplamanın hala altın standart olduğu bilinmektedir (3). Bununla birlikte, bilgisayarlı sistemler hacimleri az veya çok tahmin edebi- 
lirler. Önceki çalışmalar, tahmini greft volümü (TGV) ile intraoperatif gerçek greft ağırlığı (GGA) arasında \%5-36 gibi geniş bir yanılma payının olduğunu göstermiştir (47). Tahmini greft ağırlığını (TGA) doğru tahmin etmek ve bu hata marjını en aza indirmek için çeşitli düzeltme faktörleri ve modifiye edilmiş formüller geliştirilmiştir (7-13). Merkezimizde düzeltme faktörü olarak, Lemke ve arkadaşlarının geliştirdiği formül kullanılmaktadır (13).

Bu çalışmanın amacı canlı vericili karaciğer transplantasyonunda greftin değerlendirilmesinde bilgisayarlı tomografi (BT) ile ölçülen TGV'den korreksiyon faktörü kullantlarak hesaplanan TGA ile GGA'nın karşılaştıııması ve bu faktörün efektivitesinin araştırılmasıdır.

\section{GEREÇ ve YÖNTEM}

Ocak 2009-Temmuz 2015 tarihleri arasında 42 canlı karaciğer donörünün (20 kadın, 22 erkek; ortalama yaş, 42.23 yıl \pm 17.25 (18-52) dinamik kontrastlı hepatik BT kesitlerinden elde edilen veriler retrospektif olarak tarandı. Çalışma için kurumsal etik kurul onayı alınmıştır. Her donör, sağılıkla ilgili ve etik yoluyla karaciğer bağışına yönelik bireysel bir standart değerlendirme sürecinden geçmiştir. Yazılı hasta onamı tüm bağışçılardan alınmışır.

Donörler, karaciğer nakli amacıyla karaciğer sağ lobunun (Couinaud sınıflandırma sistemine göre V, VI, VII ve VIII segmentleri) rezeksiyonu yapılan sağlıklı yetişkinlerdi. Elde edilen 42 greftin otuz dokuzu orta hepatik veni dışlayan sağ loblardı. Kalan üç greftin orta hepatik veni de içerdiği tespit edildi.

\section{BT Protokolü}

Tüm donör adaylarına 64 kesit BT (Toshiba Aquilion), (Toshiba Medical Systems, Otawara, Japonya) cihazı ile kontrastlı dört fazıı bir tomografi tetkiki uygulandı. Kontrastsız faz ile hepatik steatoz (14) değerlendirildi. Karaciğerde \%10'dan daha fazla parankimal yağlanma bir donör dışlama kriteridir (15). Hepatik arteriyel, portal venöz ve hepatik venöz fazlardan oluşan kontrastlı fazlar vasküler yapıların üç boyutlu rekonstrüksiyonu yoluyla cerrahi olarak anatomik varyasyonları analiz etmek için kullanıldı.

Toplam 120-140 mL noniyonik kontrast madde (iopromid, Ultravist 370, Bayer Schering Pharma), bir enjektör ile $4.0 \mathrm{~mL} / \mathrm{s}$ hızında antekubital bir venden enjekte edildi. Bolus-takip tekniği ile inen aortun 180 HU'ya kadar kontrastlanmasından 5 saniye sonra arteriyel faz görüntüleme başlatıldı. Portal venöz faz ve hepatik venöz faz için gecikme süreleri, kontrast infüzyonunun başlamasından sonra sırasıyla 65 saniye ve 120 saniye idi. Hepatik venöz faz hepatik venlerin maksimum kontrastla dolduğu evre olduğu için volümetrik ölçüm bu fazda yapıldı (16).
BT görüntüleri için tarama ve rekonstrüksiyon parametreleri aşağıdaki gibidir: Kolimasyon; $64 \times 0.5 \mathrm{~mm}$, rekonstrüksiyon kalınlığı; $1 \mathrm{~mm}$, rekonstrüksiyon intervali; 0.5 $\mathrm{mm}$, pitch; 1.5, gantri rotasyon süresi, 0.6 saniye, 120 $k V p$ ve $110-320$ mAs.

\section{BT Volümetri}

BT görüntüleri çalışma istasyonu (Advanced Workstation 4.4, GE Medical System, Wisc .; $A B D$ ) üzerinde bir radyolog (10 yıllık deneyime sahip, M.Ö.) tarafından değerlendirildi. Radyolog aksiyel BT görüntüleri üzerinde tüm karaciğer alanını işaretçiyi sürükleyerek boyadı. Porta hepatis, inferior vena kava, ekstrahepatik portal venler gibi büyük damarlar, büyük fissürler ve ligamentler manuel volümetrik işaretlemenin dışında bırakıldı (Resim 1). Ölçümler bittikten sonra karaciğere 3 boyutlu volüm kazandırıldı ve total volümün kaç cc olduğu görüldü. Ardından bu görüntü sağ ve sol lobların diseksiyon hattı boyunca kesilip sağ ve sol lobların ayrı ayrı hacimleri hesaplandı. İnferior vena kavanın ortası bir oryantasyon noktası olarak kullanıldı ve orta hepatik venin sağ tarafından safra kesesi yatağına doğru kraniokaudal diseksiyon hattı çizilerek sanal kesme işlemi uygulandı (Resim 2). Sağ lob volümünün alıcıya yetmeyeceği düşünülen 3 donörde bu çizgi kraniokaudal olarak orta hepatik venin solundan yapıldı. Vericide kalacak tahmini karaciğer hacmi totalin \%30'undan az olduğu adaylar nakile uygun bulunmadı.

BT cihazının oluşturduğu TGV, intraoperatif GGA ile karşılaştırıldı. Hesaplanan TGV'den (cc) Lemke ve arkadaşlarının formülü [TGA (g): 0.678 x TGV (cc) +143.704 g] kullanılarak TGA tespit edildi (13). Greft- alıcı ağırlık oranı $\% 0.8$ 'den daha büyük olan greft nakli tercih edildi.

\section{Intraoperatif Prosedür ve Gerçek Greft Ağırlığının Ölçülmesi}

Canlı donör cerrahisinde preoperatif görüntülenen diseksiyon düzlemi boyunca parsiyel hepatektomi yapıldı. Çıkarılan karaciğer hemen $4{ }^{\circ} C^{\prime}$ de histidin-triptofan-ketoglutarat çözeltisi (Custodiol; Köhler Chemie, Alsbach-Hähnlein, Almanya) ile yıkandı. Çözelti karaciğerden tamamen boşaltıldıktan sonra GGA otomatik bir tartma makinesi ile gram cinsinden ölçüldü.

\section{İstatistiksel Analiz}

İstatistiksel analiz SPSS 21.0 sürümü (spss inc., Chicago) kullanılarak yapıldı. Tanımlayıcı veri analizi için, ameliyat öncesi ve ameliyat verilerinin dağılımı grafiksel olarak gösterildi. TGV, TGA ve GGA arasındaki ilişkinin değerlendirilmesinde Pearson korelasyon testi kullanıldı. $p<0,05$ değeri anlamlı kabul edildi. 

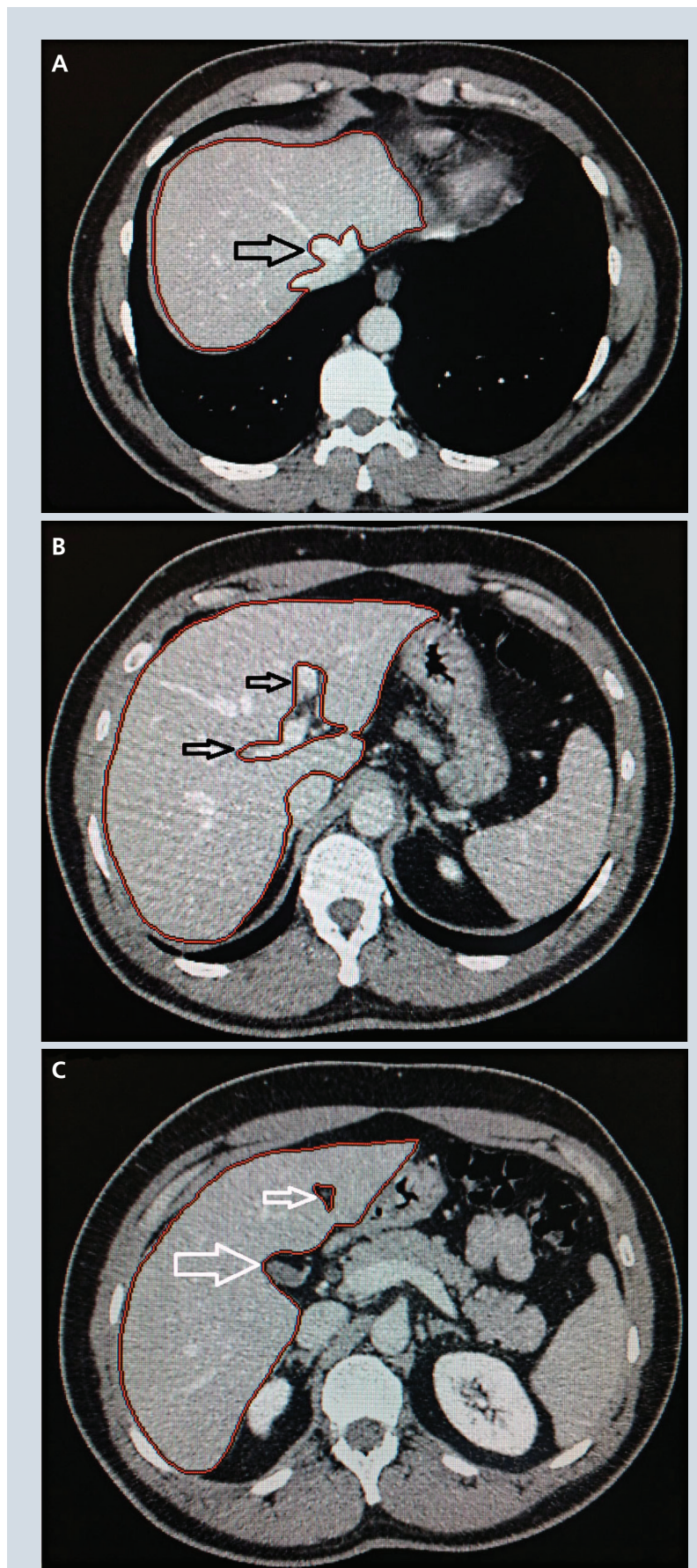

Resim 1. Manuel BT volümetri; hepatik venöz faz BT kesilerinde; (A) hepatik venlerin proksimal açılım lokalizasyonları, vena cava (büyük siyah ok), (B) sağ ve sol portal venler (küçük siyah oklar), (C) ligamentum teres ve safra kesesi (küçük ve büyük beyaz oklar) manuel ölçümün dışında bırakıımışıı.

\section{BULGULAR}

Manuel hacim ölçümü 15.6 (11-19) dakika içinde tamamlandı. Ameliyat öncesi ortalama TGV 918.265 (580-1230) cc idi. Ameliyat öncesi TGA ve intraoperatif GGA ortalama ağırlıkları sırasıyla 818.119 (550-1171) gr, 790.359 (505-1150) gr idi.

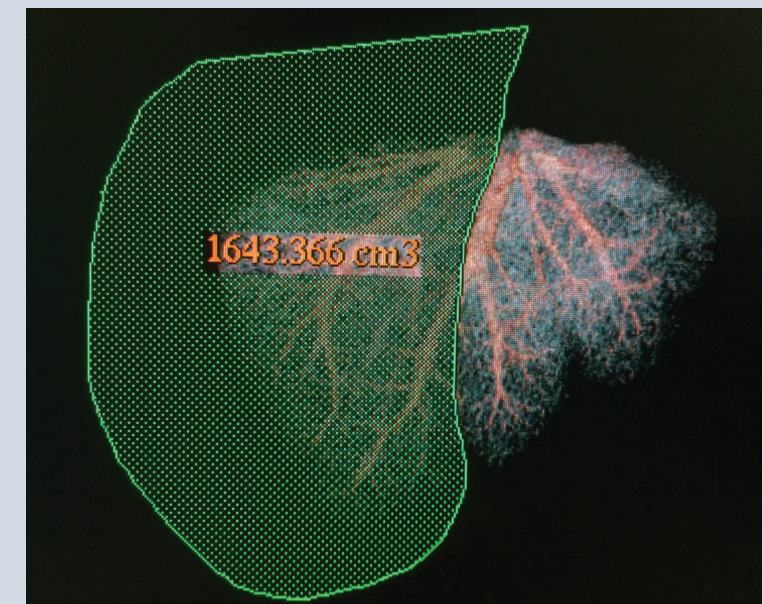

Resim 2. Volüm kazandırılmış BT imajında; total karaciğerden sağ lobun ayrıldığı diseksiyon hattı izlenmekte.

GGA ile TGV arasında istatistiksel bir ilişki yoktu. Ancak GGA ile TGA arasında güçlü bir istatistiksel ilişki bulundu. Pearson korelasyon testi bu korelasyonu gösterdi ( $r$ $=0.98, p<0.001$ ). Regresyon modelinin sonucu da bu bulguyu destekledi $(p<0.0001)$.

Transplantasyonun ardından greft- alıcı ağırlık oranı \%0.81 olan 3 hastada (\%7.1) küçük boyut sendromu ile karşılaşıldı. Bu hastalarda TGA ile GGA birbirine çok yakın değerlerdeydi. Karaciğer testi sonuçları, ameliyattan ortalama 2.5 hafta sonra normal sınırlara ulaşarak yeterli greft rejenerasyonu sonucu greftlerin hacimleri arttı.

Beş hastada (\%11.9) greft- alıcı ağırlık oranı \%3'ün üzerindeydi ve büyük boyutlu greftlerle ilişkili hiçbir cerrahi komplikasyon görülmedi. Bu hastalarda da TGA ile GGA birbirine çok yakın değerlerde ölçüldü.

\section{TARTIŞMA}

Canlı vericili karaciğer transplantasyonunda verilecek greft boyutunun doğru tahmin edilmesi donör değerlendirmesinin önemli bir bileşenidir. Bu nedenle, karaciğer transplantasyonu planlaması için doğru ve noninvaziv bir volümetrik inceleme gereklidir. BT; noninvaziv olması, yüksek uzaysal ve kontrast çözünürlüğüne sahip olması nedeni ile bu amaç için iyi bir yöntemdir $(13,17)$. BT volümetri hem donör hem de alıc için çok önemli bilgiler sağlar. Donörde kalacak karaciğer miktarı toplam karaciğer hacminin \%30'undan fazla olmalıdır (18-19). Potansiyel donörün greft boyutunu değerlendirmek için greft- alıcı ağırlık oranı kullanılmıştır. Bu değerin \%0.8'den daha düşük olması nakil sonrası mortalite ve morbidite artışı ile ilişkilendirilmiştir (18-21). Bu nedenle, preoperatif olarak beklenen greft- alıcı ağırlık oranı \%0.8'e yakın ise cerrah- 
lar tahmini ağırlığı daha yüksek doğrulukla bilmek isterler. Preoperatif BT görüntüleme ile greft ağırlığının TGV'den kesin olarak tahmin edilmesi büyük önem taşır $(22,23)$.

BT volümetrik inceleme hem manuel hem de otomatik ölçümlerle karaciğerin cc olarak hacim değerini vermektedir (3). Karaciğer hacminin hesaplanması için otomatik yazılımın piyasaya sürülmesi ile hacimsel değerlendirme çok daha az zaman alıcı hale gelmiş olmakla birlikte klinik uygulamada hala eksik yönleri bulunmaktadır (24). Yeniliklere ve yardım programlarının kullanılmasına rağmen, karaciğer sınıının BT görüntülerinde manuel olarak elle çizilmesi, karaciğer hacminin hesaplanmasında hala altın standart tekniktir (3).

1985 yılında Van Thiel ve arkadaşları klasik olarak BT ile ölçülen $1 \mathrm{~mL}$ karaciğer hacminin 1 gr karaciğer ağırlığına eşit olduğunu kabul etmişti (25). Ancak daha sonra çeşitli araştırmalarda cc olarak ölçülen preoperatif TGV'nin gram olarak ölçülen GGA'dan daha büyük olduğunu ve bu ikili arasında $\pm \% 5$ ile $\pm \% 36$ arasında yanılma payının olabileceği bildirilmiştir $(10,13,26-29)$. Bu yanılma payının intraoperatif kanın drenajı, anatomik varyasyon, interobserver varyasyon ve BT cihazı varyasyonu gibi çeşitli nedenleri olduğu düşünülmüştür. Nedenden bağımsız olarak BT ile ölçülen volümü tahmini grama çeviren çeşitli formüller ve düzeltme faktörleri tanımlanmıştır $(7,11,13,26,30)$. Bunlardan birisi de Lemke ve arkadaşlarının sağ lob greftlerinde geliştirdiği formüldür. Bu çalışmada hasta sayısının az olmasına rağmen ( $n: 16)$ tahmini ağırlık ile aktüel gram arasında korelasyon olduğu bildi-

\section{KAYNAKLAR}

1. Mazzaferro V, Regalia E, Doci R, et al. Liver transplantation for the treatment of small hepatocellular carcinomas in patients with cirrhosis. $\mathrm{N}$ Engl J Med 1996;334:693-700.

2. Trotter JF, Wachs M, Everson GT, Kam I. Adult-to-adult transplantation of the right hepatic lobe from a living donor. $N$ Engl J Med 2002;346:1074-82.

3. Suzuki K, Epstein ML, Kohlbrenner R, et al. Quantitative radiology: automated CT liver volumetry compared with interactive volumetry and manual volumetry. AJR Am J Roentgenol 2011;197:W706-12.

4. Morgan G, Diflo T, John D, Fryer J, Teperman L. Selection and imaging of the living liver donor. Curr Opin Organ Transplant 2001;6:350-4.

5. Fulcher A, Szucs R, Bassignani M, Marcos A. Right lobe living donor liver transplantation: Preoperative evaluation of the donor with MR imaging. AJR Am J Roentgenol 2001;176:1483-5.

6. Schroeder T, Malago M, Debatin JF, et al. All-in one imaging protocols for the evaluation of potential living liver donors: Comparison of magnetic resonance imaging and multidetector computed tomography. Liver Transpl 2005;11:776-87.

7. Salvalaggio $P$, Baker $T$, Koffron $A$, et al. Liver graft volume estimation in 100 living donors: Measure twice, cut once. Transplantation 2005;80:1181-5. rilmiştir (13). Bizim çalışmamızda da kliniğimizde rutin olarak kullandığımız Lemke ve arkadaşlarının formülünün aktüel gram tahmininde etkili olduğu istatistiksel olarak tespit edildi $(r=0.98, p<0.001)$.

Bu çalışmanın birinci limitasyonu, Lemke ve arkadaşlarının çalışmasından daha fazla olmasına rağmen hasta sayısının yetersizliği idi. İkinci limitasyon, manuel hacimlerin tek bir radyolog tarafından ölçülmesiydi. Manuel hacimlerin karaciğer transplantasyonunda deneyimli birkaç uzman radyolog tarafından ölçülmesi ideal olarak kabul edilir ancak bu değerlendirme tüm kurumlarda karaciğer nakli konusunda yeterince deneyimli radyolog bulunmadığı için her zaman mümkün olmaz. Bu çalışmada ölçümler karaciğer nakli konusunda 10 yıldan fazla deneyime sahip olan bir radyolog tarafından yapılmıştır. Tecrübesiz radyologlar ve/veya tecrübeli ve tecrübesiz radyolog birlikteliği ile yapılan ölçümlerin güvenilirliğinin daha az olacağını düşünmekteyiz. Ayrıca manuel BT volümetri ölçümlerinde iki tecrübeli radyolog arasında 0.997 gibi çok yüksek oranda interobserver korelasyon olduğunu belirten bir çalışma da mevcuttur (31).

Sonuç olarak canlıdan karaciğer naklinde greftin değerlendirilmesinde BT ile ölçülen volümün gerçek ağırlıktan farklı olduğu, Lemke ve arkadaşlarının geliştirdiği formülün aktüel ağırığı tahminde yeterli ve etkili olduğu tespit edildi. Bu formülün klinik kullanıma daha yaygın olarak girmesi için diğer formüllerle karşılaştırılarak daha fazla hasta sayısı ile geniş çaplı araştırmalar yapılmalıdır.

8. Lemke AJ, Brinkmann MJ, Pascher A, et al. Accuracy of the CT-estimated weight of the right hepatic lobe prior to living liver donation (LRLD) for predicting the intraoperatively measured weight of graft.Rofo 2003;175:1232-8.

9. Yoshizumi T, Gondolesi C, Bodian $\mathrm{H}$, et al. A simple new formula to assess liver weight. Transplant Proc 2003;35:1415-20.

10. Kim KW, Lee J, Lee $H$, et al. Right lobe estimated bloodfree weight for living donor liver transplantation: accuracy of automated blood-free CT volumetry-preliminary results. Radiology 2010;256:43340.

11. Yoneyama T, Asonuma K, Okajima H, et al. Coefficient factor for graft weight estimation from preoperative computed tomography volumetry in living donor liver transplantation. Liver Transpl 2011;17:369-72.

12. Mokry T, Bellemann N, Müller D, et al. Accuracy of estimation of graft size for living-related liver transplantation: first results of a semi-automated interactive software for CT-volumetry. PLoS One 2014;9:e110201.

13. Lemke AJ, Brinkmann MJ, Schott T, et al. Living donor right liver lobes: preoperative CT volumetric measurement for calculation of intraoperative weight and volume. Radiology 2006;240:73642. 
14. Limanond P, Raman SS, Lassman C, et al. Macrovesicular hepatic steatosis in living related liver donors: correlation between CT and histologic findings. Radiology 2004;230:276-80.

15. Steinmuller $T$, Pascher A, Sauer $I M$, et al. Living donor liver transplantation of the right liver lobe between adults [in German]. Dtsch Med Wochenschr 2002;127:1067-71.

16. Schroeder T, Nadalin S, Stattaus J, et al. Potential living liver donors: evaluation with an all-in-one protocol with multi-detector row CT. Radiology 2002;224:586-91.

17. Emiroglu R, Coskun M, Yilmaz U, et al. Safety of multidetector computed tomography in calculating liver volume for living-donor liver transplantation. Transplant Proc 2006;38:3576-8.

18. Akabayashi A, Slingsby BT, Fujita M. The first donor death after living-related liver transplantation in Japan. Transplantation 2004; $77: 634$

19. Kamel IR, Kruskal JB, Warmbrand G, et al. Accuracy of volumetric measurements after virtual right hepatectomy in potential donors undergoing living adult liver transplantation. Am J Roentgenol 2001;176:483-7.

20. Kiuchi T, Kasahara M, Uryuhara K, et al. Impact of graft size mismatching on graft prognosis in liver transplantation from living donors. Transplantation 1999;67:321-7.

21. Lo CM. Complications and long-term outcome of living liver donors: a survey of 1,508 cases in five Asian centers. Transplantation 2003;75(3 Suppl):S12-5.

22. Heaton N. Small-for-size liver syndrome after auxiliary and split liver transplantation: donor selection. Liver Transpl 2003;9:S26-8.
23. Kiuchi T, Tanaka K, Ito T, et al. Small-for-size graft in living donor liver transplantation: how far should we go? Liver Transpl 2003;9:S29-35.

24. Luciani A, Rusko L, Baranes L, et al. Automated liver volumetry in orthotopic liver transplantation using multiphase acquisitions on MDCT. AJR Am J Roentgenol 2012;198:W568-74.

25. Van Thiel DH, Hagler NG, Schade RR, et al. In vivo hepatic volume determination using sonography and computed tomography. Gastroenterology 1985;88:1812-7.

26. Hwang S, Lee SG, Kim KH, , et al. Correlation of blood-free graft weight and volumetric graft volume by an analysis of blood content in living donor liver grafts. Transplant Proc 2002;34:3293-4.

27. Gondolesi GE, Yoshizumi T, Bodian C, et al. Accurate method for clinical assessment of right lobe liver weight in adult living-related liver transplant. Transplant Proc 2004;36:1429-33.

28. Radtke A, Sotiropoulos GC, Nadalin S, et al. Preoperative volume prediction in adult living donor liver transplantation: how much can we rely on it? Am J Transplant 2007;7:672-9.

29. Yuan D, Chen K, Li B, et al. Accurate and reasonable method for estimation of graft volume in living donor liver transplantation. Transplantation 2008;86:1011-2.

31. Sandrasegaran K, Kwo PW, DiGirolamo D, et al. Measurement of liver volume using spiral CT and the curved line and cubic spline algorithms: reproducibility and interobserver variation. Abdom Imaging 1999;24:61-5. 